\title{
Pearls
}

\section{Pearls: The Mobile Segment in Sarcoma Resections}

\author{
R. Lor Randall MD, FACS
}

ک he classic teaching of distal femoral resection for bone sarcomas dictates that dissection and resection should occur in situ. Generally, a medial approach is recommended in order to dissect out the vessels through Hunter's canal [1]. However, some tumors present with a larger extraosseous component laterally, and when this occurs, surgeons may perform a laterally based biopsy. Thus, at the time of resection, the lateral biopsy tract (if done open) should be resected while a medial approach

\section{A note from the Editor-in-Chief:}

We are pleased to present the next installment of "Pearls", a column in Clinical Orthopaedics and Related Research ${ }^{\circledR}$. In this column, distinguished surgeons, scientists, or scholars share surgical or professional tips they use to help surmount important or interesting problems. We welcome reader feedback on all of our columns and articles; please send your comments to eic@clinorthop.org.

The author certifies that neither he, nor any members of his immediate family, have any commercial associations (such as consultancies, stock ownership, equity interest, patent/licensing arrangements, etc) that might pose a conflict of interest in connection with the submitted article. All ICMJE Conflict of Interest Forms for authors and Clinical Orthopaedics and Related Research ${ }^{\mathbb{R}}$ editors and board members are on file with the publication and can be viewed on request. for resection is performed. Here, I propose a different approach (or philosophy) to sarcoma resection; a concept dubbed "the mobile segment".

When I first started practice, I rarely had residents or assistants during surgery, and so I often performed sarcoma resections exclusively with a surgical technician. Because of this, I needed to come up with a technique that enabled the soft tissues to fall away as I dissected structures away from the bone and tumor. The mobile-segment approach provides this.

The steps are relatively straightforward. After making the skin incision, and measuring the resection length from the joint, I dissect down to the bone at that level of the osteotomy and make my cut. Next, I apply bone wax and place a large bone-reduction clamp on the end of the bone to be resected. After confirming that the marrow margin is free of tumor based

The opinions expressed are those of the writers, and do not reflect the opinion or policy of $C O R R^{\circledR}$ or The Association of Bone and Joint Surgeons ${ }^{\mathrm{R}}$.

R. L. Randall MD, FACS ( $₫)$

Huntsman Cancer Institute, 2000 Circle of Hope Dr, Salt Lake City, UT 84112, USA

e-mail: lor.randall@hci.utah.edu upon frozen section, I develop my "mobile segment" back towards the tumor and the joint. The scrub tech applies gentle tension with one hand on the forceps while retrieving instruments with the other. I only need a little additional traction to carry out the rest of the resection without a problem (Fig. 1).

Even if one has additional assistants in the operating room, I find that the mobile segment technique simplifies the dissection, making it faster and safer; less surgical time means less time under anesthesia, and perhaps a lower risk of infection.

This technique also makes the approach to the extremity universal in terms of vascular access and dissection. All variables being equal, I prefer to do a distal femoral resection laterally. The vastus lateralis is far more forgiving when rehabilitating the extensor mechanism, and the approach enables straightforward proximal extension to a total femoral replacement. With a mobile segment established, it is also easy to access the vessels from a lateral approach.

I use a mobile segment for all of my sarcoma resections, including those of soft tissue. When dealing with softtissue sarcomas, I orient the dissection so as to find a portion of the tumor that 


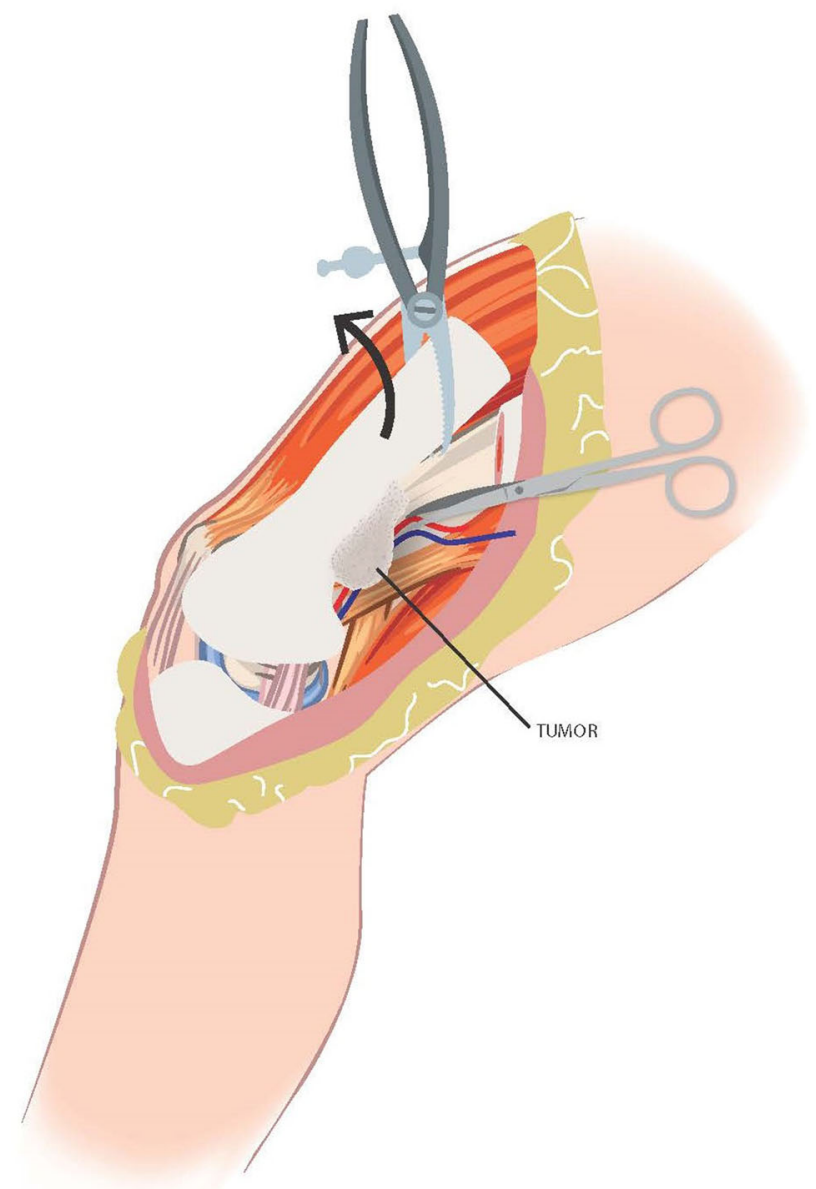

Fig. 1 A schematic representation of a distal femoral resection using a mobile segment is shown. The bone is osteotomized at the diaphysial level of resection and a bone clamp is applied. The clamp is then used to apply gentle traction while dissection is carried out (scissors) towards the joint. By using this technique, the critical structures fall away facilitating dissection and improving the oncologic margin. (Published with permission from Courtney Colvin, Huntsman Cancer Institute).
I can mobilize with relative ease, and then removal of the remainder of the tumor then follows without much difficulty. As gentle traction is applied to the mass and its corresponding margin, the neurovascular bundle falls away as I dissect them free, allowing me to remove the sarcoma.

\section{Reference}

1. Malawer MM. Distal femoral resection with endoprosthetic reconstruction. In: Malawer MM, Sugarbaker PH, eds. Musculoskeletal Cancer Surgery: Musculoskeletal Cancer Surgery Treatment of Sarcomas and Allied Diseases. Dordrecht, the Netherlands: Kluwer Academic Publishers; 2001:457. 